\title{
Indirect CP violation at Belle
}

\author{
Bastian Kronenbitter* \\ KIT \\ E-mail: kronenbitter@ekp.uni-karlsruhe.de
}

We present a summary of the measurements of indirect $C P$ and $C P T$ violation, which were performed by the Belle collaboration using data samples of up to $772 \times 10^{6} B \bar{B}$ pairs, collected at the $\Upsilon(4 S)$ resonance with the Belle detector at the KEKB asymmetric-energy $e^{+} e^{-}$collider.

These measurements include the determination of time-dependent $C P$ violation in a variety of decays of neutral $B$ mesons to $C P$ eigenstates, but also the measurement of $C P$ violation in the decays of $B$ mesons at the $\Upsilon(5 S)$ resonance with a new tagging algorithm. We also present the most precise measurement of $C P T$ violation in the neutral $B$ system.

36th International Conference on High Energy Physics

4-11 July 2012

Melbourne, Australia

${ }^{*}$ Speaker. 


\section{Introduction}

One of the main goals of the Belle experiment is the measurement of $C P$ violation and constraining the unitarity triangle. Especially the measurement of time-dependent $C P$ violation is one of the strong points of $B$ factories and their special structure allows for a high precision and broad range of analyses of time-dependent phenomena.

The decay $B^{0} \rightarrow(c \bar{c}) K^{0}$ is commonly known as the golden channel of time-dependent $C P$ violation and in this decay channel, the first observation of mixing-induced $C P$ violation could be achieved. While the first measurements in this decay channel were performed more than ten year ago, the largely increased data set of $770 \times 10^{6} B \bar{B}$ pairs now allows both for a great precision in this decay channel and for the measurement of time-dependent $C P$ violation in other decay channels like $B^{0} \rightarrow D^{(*)+} D^{(*)-}$.

With the Belle experiment having stopped data taking and the new and improved BelleII experiment expected to be starting in 2016, the development and testing of new methods is especially interesting. One of them is the measurement of $C P$ violation in $\Upsilon(5 S)$ events, using a new tagging method, which also could be used to measure the $C P$ violation in decays of $B$ mesons to $C P$ eigenstates without charged tracks in the final state.

\section{2. $C P$ violation in $B^{0} \rightarrow(c \bar{c}) K^{0}$ decays [1]}

We reconstruct these decays in five different decay modes, listed in Table 1. Signal candidates are selected based on various properties like the mass of intermediate particles or particle identification information, provided by different sub-detectors. The probability of a candidate to be a correctly reconstructed signal candidate is determined with fits in different observables. In case of $B^{0} \rightarrow J / \psi K_{L}$, where the $K_{L}$ cannot be detected due to its long lifetime, the fit is performed using the momentum of the reconstructed $B$ meson in the center-of-mass system $p_{B}^{*}$. In all other decay channels the mass of the $B$ meson $M_{\mathrm{bc}}$ is used, where the measured energy is replaced with the well known beam energy.

\begin{tabular}{lcc}
\hline Decay mode & \multicolumn{1}{c}{$N_{\text {sig }}$} & Purity (\%) \\
\hline$J / \psi K_{S}^{0}$ & $12649 \pm 114$ & 97 \\
$\psi(2 S)\left(\ell^{+} \ell^{-}\right) K_{S}^{0}$ & $904 \pm 31$ & 92 \\
$\psi(2 S)\left(J / \psi \pi^{+} \pi^{-}\right) K_{S}^{0}$ & $1067 \pm 33$ & 90 \\
$\chi_{c 1} K_{S}^{0}$ & $940 \pm 33$ & 86 \\
$J / \psi K_{L}^{0}$ & $10040 \pm 154$ & 63 \\
\hline
\end{tabular}

Table 1: Decay channels reconstructed in the $B^{0} \rightarrow(c \bar{c}) K^{0}$ analysis.

The decay time difference of the two $B$ mesons is extracted using their decay vertex information, which is determined with a series of kinematic fits. The flight length difference $\Delta z$ is directly related to the decay time difference $\Delta t$ by the boost of the center-of-mass system, which is given by the energy asymmetry of the electron and positron beams. The tracks not used for the reconstruction of the signal $B$ meson are taken to determine the flavor of the accompanying $B$ meson, $q$. 
This information describes the time-dependent decay rate of the neutral $B$ meson with

$$
\mathscr{P}(\Delta t)=\frac{1}{4 \tau_{B_{d}}} e^{-|\Delta t| / \tau_{B_{d}}}\{1+q[\mathscr{S} \sin (\Delta m \Delta t)+\mathscr{A} \cos (\Delta m \Delta t)]\},
$$

where $\mathscr{S}$ is related to mixing-induced $C P$-violation, $\mathscr{A}$ is related to direct $C P$-violation, and $\tau_{B_{d}}$ is the $B$ lifetime.

The according fit is illustrated in Fig. 1 and gives $\mathscr{S}=0.667 \pm 0.023$ (stat.) \pm 0.012 (syst.) and $\mathscr{A}=0.005 \pm 0.016$ (stat.) \pm 0.012 (syst.).

As $\mathscr{S}$ is directly related to the angle $\phi_{1}$ of the unitarity triangle with $\mathscr{S}=\sin 2 \phi_{1}$, this leads to the most precise single determination of a CKM-angle. The increase in precision in comparison to the last Belle measurement [2] could be reached not only due to the bigger data sample, but also due to the reprocessing of a significant fraction of the data using new track finding algorithms and the introduction of a new vertex-reconstruction goodness-of-fit description.
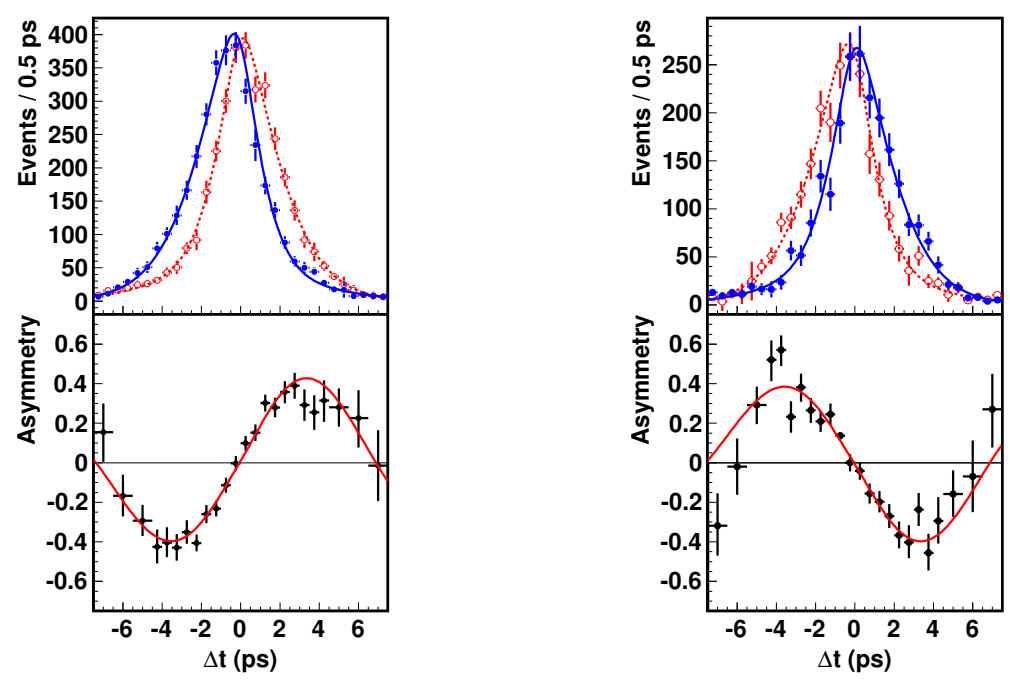

Figure 1: On the left (right): $\Delta t$ distribution of $C P$-even ( $C P$-odd) final states. The red (blue) line and markers show the data points and the corresponding fit of events for $q=1(q=-1)$.

\section{3. $C P$-violation in double charm decays $[3,4]$}

The determination of the $C P$ violation in decays of a neutral $B$ meson to two charged $D$ mesons is in many aspects very similar to the analysis of $B^{0} \rightarrow(c \bar{c}) K^{0}$ decays. Also here one $B$ meson is fully reconstructed and selected based on information of the intermediate particles. The signal probability also is determined with a fit on $M_{\mathrm{bc}}$ and additionally the difference between the nominal and reconstructed energy $\Delta E$.

But each of the three studied decay channels $B^{0} \rightarrow D^{+} D^{-}, B^{0} \rightarrow D^{* \pm} D^{\mp}$, and $B^{0} \rightarrow D^{*+} D^{*-}$ has specific features, which need to be taken care of.

$B^{0} \rightarrow D^{+} D^{-}$: This decay showed huge direct $C P$-violation in the last measurement of the Belle collaboration, which is not expected by the standard model [5]. The presence of a large amount 
of background, consisting of wrongly reconstructed $e^{+} e^{-} \rightarrow(q \bar{q}), q \in\{u, d, s, c\}$ continuum events makes it necessary to suppress this kind of background. This is achieved with information about the event topology, like Fox-Wolfram moments, which are combined using a neural network.

$B^{0} \rightarrow D^{* \pm} D^{\mp}$ : This is not a decay to a $C P$ eigenstate. Therefore the description of the $\Delta t$ distribution has to be modified to include the distinction between $B^{0} \rightarrow D^{*+} D^{-}$and $B^{0} \rightarrow D^{+} D^{*-}$ decays. This is done by introducing three new variables: The parameter $A$ describes the asymmetry of the total number of the events and $\Delta \mathscr{S}$ and $\Delta \mathscr{A}$ are related to the asymmetry in $\mathscr{S}$ and $\mathscr{A}$, respectively.

$B^{0} \rightarrow D^{*+} D^{*-}$ : This is a decay to the sum of $C P$-even and $C P$-odd final states, depending on the relative angular momentum of the two $D^{*}$ mesons. The probability of an event to be $C P$-even is extracted with an angular analysis, using two of the three possible angles in the transversity base. The fraction of the $C P$-odd component $R_{\perp}$, as well as of the longitudinal component $R_{0}$ is determined together with $\mathscr{S}$ and $\mathscr{A}$ in a five-dimensional fit in $M_{\mathrm{bc}}, \Delta E, \Delta t$, and the two angles.

This fit is performed under the assumption that the $C P$ parameters for the $C P$-even and $C P$-odd states are the same, but this assumption can be release by introducing different $\mathscr{A}$ and $\mathscr{S}$ values for the two final states. This is done additionally to the nominal fit.

The results of all the measurements are listed in Table 2 and 3. All of them are consistent with the standard model expectation of $\mathscr{S}=\sin 2 \phi_{1}$ and $\mathscr{A} \approx 0$, where the measurement in $B^{0} \rightarrow D^{*+} D^{*-}$ is the first measurement, which exhibits $C P$ violation with a significance greater than $5 \sigma$.

\begin{tabular}{lrrr}
\hline decay channel & \multicolumn{1}{c}{$N_{\text {sig }}$} & \multicolumn{1}{c}{$\mathscr{S}$} & \multicolumn{1}{c}{$\mathscr{A}$} \\
\hline$B^{0} \rightarrow D^{+} D^{-}$ & $269 \pm 21$ & $-1.06_{-0.14}^{+0.21} \pm 0.08$ & $0.43 \pm 0.16 \pm 0.05$ \\
$B^{0} \rightarrow D^{* \pm} D^{\mp}$ & $887 \pm 39$ & $-0.78 \pm 0.15 \pm 0.05$ & $0.01 \pm 0.11 \pm 0.04$ \\
$B^{0} \rightarrow D^{*+} D^{*-}$ & $1225 \pm 58$ & $-0.79 \pm 0.14 \pm 0.03$ & $0.15 \pm 0.08 \pm 0.02$ \\
$B^{0} \rightarrow\left(D^{*+} D^{*-}\right)_{C P-\text { even }}$ & & $-0.81 \pm 0.13 \pm 0.03$ & $0.18 \pm 0.10 \pm 0.05$ \\
$B^{0} \rightarrow\left(D^{*+} D^{*-}\right)_{C P-\text { odd }}$ & & $1.52 \pm 0.62 \pm 0.12$ & $-0.05 \pm 0.39 \pm 0.08$ \\
\hline
\end{tabular}

Table 2: Results of common parameters of the $B^{0} \rightarrow D^{(*)+} D^{(*)-}$ measurements with the first and second error always being statistical and systematic respectively.

$$
\begin{array}{lr}
A_{D^{* \pm} D^{\mp}}= & 0.06 \pm 0.05 \pm 0.02 \\
\Delta \mathscr{S}_{D^{* \pm} D^{\mp}}= & -0.13 \pm 0.15 \pm 0.04 \\
\Delta \mathscr{A}_{D^{* \pm} D^{\mp}}= & 0.12 \pm 0.11 \pm 0.03 \\
R_{0 D^{*+} D^{*-}}= & 0.62 \pm 0.03 \pm 0.01 \\
R_{\perp D^{*+} D^{*-}}= & 0.14 \pm 0.02 \pm 0.01
\end{array}
$$

Table 3: Results of decay channel specific parameters of the $B^{0} \rightarrow D^{(*)+} D^{(*)-}$ measurements with the first and second error always being statistical and systematic respectively.

\section{Measurement of $\sin 2 \phi_{1}$ in $\Upsilon(5 S)$ decays [6]}

This measurement is performed using the final Belle $\Upsilon(5 S)$ data sample of $121 \mathrm{fb}^{-1}$. It exploits the fact, that unlike at the $\Upsilon(4 S)$ resonance, not only a pair a $B$ mesons can be produced, but 
additionally a pion. In a decay like $\Upsilon(5 S) \rightarrow \bar{B}^{(*) 0} B^{(*)+} \pi^{-}$the flavor of the neutral $B$ meson can be extracted by measuring the charge of the accompanying pion. From the asymmetry between the flavors $A_{B B \pi}$, the $C P$ violation in these decays can be extracted with

$A_{B B \pi}=\frac{N_{B B \pi^{-}}-N_{B B \pi^{+}}}{N_{B B \pi^{-}}+N_{B B \pi^{+}}}=\frac{\mathscr{S} x+\mathscr{A}}{1+x^{2}}$,

where $x=\Delta m_{d} / \Gamma_{d}$.

In this analysis, the neutral $B$ meson is reconstructed in the $C P$ eigenstate $B^{0} \rightarrow J / \psi K_{S}$ and combined with a charged track. In that way, $75.9 \pm 9.5$ neutral $B$ mesons could be reconstructed. The charged $B$ mesons are not explicitly reconstructed but their number is extracted with a fit on the missing invariant mass. The data distribution and fits for both of the sample with positively and negatively charged accompanying pion are shown in Figure 2.
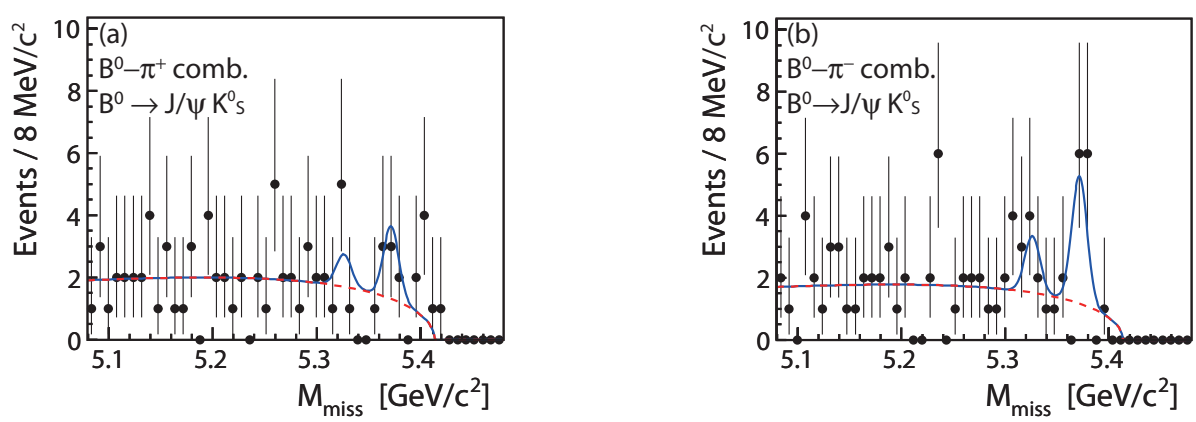

Figure 2: Data points and fitted distribution of the missing mass in $\Upsilon(5 S)$ events, where a reconstructed $B^{0}$ meson is combined with a pion candidate. The left (right) plot shows events with a positively (negatively) charged pion.

These fits give $N_{B B \pi^{+}}=7.8 \pm 3.9$ and $N_{B B \pi^{-}}=13.7 \pm 5.3$, thus $A_{B B \pi}=0.28 \pm 0.28$. Assuming $\mathscr{A}=0$, the measured value of $\sin 2 \phi_{1}$ can be extracted with

$$
\sin 2 \phi_{1}=0.57 \pm 0.58 \text { (stat.) } \pm 0.06 \text { (syst.). }
$$

This result may not be comparable with the precision reached in the measurements at the $\Upsilon(4 S)$ resonance, but it illustrates the possibility of the measurements of $C P$ violation in completely new decay channels. Also no information about the decay time of the $B$ mesons is necessary, therefore the systematic errors are independent of the ones of the measurements at the $\Upsilon(4 S)$ resonance.

\section{Measurement of $C P T$ violation [7]}

The possibility of $C P T$ violation can be included in the description of $B$ decays, although it is not part of the standard model. The time dependent decay rate of neutral $B$ mesons to final states of different kinds is described with

$$
\begin{aligned}
\mathscr{P}(\Delta t)=\frac{1}{2 \tau_{B_{d}}} e^{-|\Delta t| / \tau_{B_{d}}\left[\frac{\left|\eta_{+}\right|^{2}+\left|\eta_{-}\right|^{2}}{2}\right.} \cosh \left(\frac{\Delta \Gamma_{d}}{2} \Delta t\right)-\mathscr{R} e\left(\eta_{+}^{*} \eta_{-}\right) \sinh \left(\frac{\Delta \Gamma_{d}}{2} \Delta t\right) \\
\left.+\frac{\left|\eta_{+}\right|^{2}-\left|\eta_{-}\right|^{2}}{2} \cos (\Delta m \Delta t)+\mathscr{I} m\left(\eta_{+}^{*} \eta_{-}\right) \sin (\Delta m \Delta t)\right],
\end{aligned}
$$


where $\Delta \Gamma_{d}$ is the decay width difference of the two $C P$ eigenstates in the $B_{d}$ system, $\eta_{+}=$ $A_{B^{0} \rightarrow f_{\mathrm{rec}}} A_{\bar{B}^{0} \rightarrow f_{\mathrm{tag}}}-A_{\bar{B}^{0} \rightarrow f_{\mathrm{rec}}} A_{B^{0} \rightarrow f_{\mathrm{tag}}}$, and $\eta_{-}=\sqrt{1-z^{2}}\left(\frac{p}{q} A_{B^{0} \rightarrow f_{\mathrm{rec}}} A_{B^{0} \rightarrow f_{\mathrm{tag}}}-\frac{q}{p} A_{\bar{B}^{0} \rightarrow f_{\mathrm{rec}}} A_{\bar{B}^{0} \rightarrow f_{\mathrm{tag}}}\right)+$ $z\left(A_{B^{0} \rightarrow f_{\text {rec }}} A_{\bar{B}^{0} \rightarrow f_{\text {tag }}}+A_{\bar{B}^{0} \rightarrow f_{\text {rec }}} A_{B^{0} \rightarrow f_{\text {tag }}}\right)$ (with $A_{B^{0} / \bar{B}^{0} \rightarrow f_{\text {rec } / \text { tag }}}$ being the decay amplitudes of the according $B$ meson to the given final state). The complex parameter $z$ describes $C P T$ violation, with a non-zero absolute value meaning the presence of $C P T$ violation.

For this measurement a data set of $535 \times 10^{6} B \bar{B}$ pairs is analyzed and one $B$ meson reconstructed in eight different decay channels. They are listed in Tab. 4 and grouped in three kinds of final states, with each of them being sensitive to different physics parameters or being necessary for the calibration of experimental techniques, like flavor tagging.

\begin{tabular}{lccl}
\hline$B$ decay mode & $N_{\mathrm{ev}}$ & Purity $(\%)$ & Sensitivity \\
\hline$J / \psi K_{S}$ & 7713 & 97.0 & \multirow{2}{*}{ Mainly $\mathscr{R} e(z)$ and $\Delta \Gamma_{d}$} \\
$J / \psi K_{L}$ & 10966 & 59.2 & \\
\hline$D^{-} \pi^{+}$ & 39366 & 83.2 & \\
$D^{*-} \pi^{+}$ & 46292 & 81.5 & Mainly $\mathscr{I} m(z)$ and flavor tagging quality \\
$D^{*-} \rho^{+}$ & 45913 & 66.3 & \\
$D^{*-} \ell^{+} v_{\ell}$ & 383818 & 75.2 & \\
\hline$J / \psi K^{+}$ & 32150 & 97.3 & \multirow{2}{*}{ Resolution parameters } \\
$\bar{D}^{0} \pi^{+}$ & 216605 & 63.9 & \\
\hline
\end{tabular}

Table 4: List of decay channels, which are reconstructed for the measurement of $C P T$ violation.

All information necessary for the fit to the $\Delta t$ distribution are extracted very similar to the measurement of $C P$ violation in $B^{0} \rightarrow(c \bar{c}) K^{0}$ decays. The according fit gives

$$
\begin{aligned}
\mathscr{R} e(z) & =(1.9 \pm 3.7(\text { stat. }) \pm 3.3(\text { syst. })) \times 10^{-2} \\
\mathscr{I} m(z) & =(-5.7 \pm 3.3 \text { (stat. }) \pm 3.3 \text { (syst. })) \times 10^{-3} \\
\Delta \Gamma_{d} / \Gamma_{d} & =(-1.7 \pm 1.8 \text { (stat. }) \pm 1.1 \text { (syst. })) \times 10^{-2},
\end{aligned}
$$

thus no significant $C P T$ violation could be observed. This is the most precise single measurement of these parameters in the neutral $B$ meson system to date.

\section{References}

[1] I .Adachi et al. (Belle collaboration), Phys. Rev. Lett. 108, 171802 (2012)

[2] K.-F. Chen et al. (Belle Collaboration), Phys. Rev. Lett. 98, 031802 (2007)

[3] M. Roehrken et al. (Belle collaboration), Phys. Rev. D 85, 091106(R) (2012)

[4] B. Kronenbitter et al. (Belle collaboration), Phys. Rev. D 86, 071103(R) (2012)

[5] S. Fratina et al. (Belle collaboration), Phys. Rev. Lett. 98, 221802 (2007)

[6] Y. Sato et al. (Belle Collaboration), Phys. Rev. Lett. 108, 171801 (2012)

[7] T. Higuchi et al. (Belle collaboration), Phys. Rev. D 85, 071105(R) (2012) 